\title{
Chronic alcohol ingestion induces osteoclastogenesis and bone loss through IL-6 in mice
}

\author{
Jinlu Dai, ${ }^{1}$ Dinlii Lin, ${ }^{1}$ Jian Zhang, ${ }^{2}$ Paula Habib, ${ }^{1}$ Peter Smith, ${ }^{1}$ Jill Murtha, ${ }^{1,2}$ Zheng Fu, ${ }^{3}$ \\ Zhi Yao, ${ }^{4}$ Yinghua Qi, ${ }^{5}$ and Evan T. Keller ${ }^{1,2,3,6,7}$ \\ ${ }^{1}$ Unit for Laboratory Animal Medicine, \\ ${ }^{2}$ Department of Pathology, and \\ ${ }^{3}$ Program in Immunology, University of Michigan, Ann Arbor, Michigan, USA, \\ ${ }^{4}$ Department of Immunology, Tianjin Medical University, Tianjin, China, \\ ${ }^{5}$ Laboratory of Cellular and Molecular Biology, Department of Internal Medicine, \\ Tianjin 3rd Municipal Hospital, Tianjin, China \\ ${ }^{6}$ Institute of Gerontology, and \\ ${ }^{7}$ Program in Comparative Integrative Genomics, University of Michigan, Ann Arbor, Michigan, USA \\ Address correspondence to: Evan T. Keller, Room 5304 CCGCB Box 0940, \\ 1500 E. Medical Center Drive, Ann Arbor, Michigan 48109-0940, USA. \\ Phone: (734) 615-0280; Fax: (734) 936-9220; E-mail: etkeller@umich.edu.
}

Received for publication June 1, 2000, and accepted August 21, 2000.

To investigate the role of IL- 6 in alcohol-mediated osteoporosis, we measured a variety of bone remodeling parameters in wild-type $\left(i l 6^{+/}\right)$or IL-6 gene knockout $\left(i l 6^{-/-}\right)$mice that were fed either control or ethanol liquid diets for 4 months. In the $i l 6^{+/+}$mice, ethanol ingestion decreased bone mineral density, as determined by dual-energy densitometry; decreased cancellous bone volume and trabecular width and increased trabecular spacing and osteoclast surface, as determined by histomorphometry of the femur; increased urinary deoxypyridinolines, as determined by ELISA; and increased CFU-GM formation and osteoclastogenesis as determined ex vivo in bone marrow cell cultures. In contrast, ethanol ingestion did not alter any of these parameters in the $i l 6^{-/-}$mice. Ethanol increased receptor activator of NF- $\mathrm{KB}$ ligand (RANKL) mRNA expression in the bone marrow of $i \mathrm{l}^{+/+}$but not $i \mathrm{l}^{-/-}$mice. Additionally, ethanol decreased several osteoblastic parameters including osteoblast perimeter and osteoblast culture calcium retention in both $i \mathrm{l}^{+/+}$and $i \mathrm{l}^{-/-}$mice. These findings demonstrate that ethanol induces bone loss through IL-6. Furthermore, they suggest that IL- 6 achieves this effect by inducing RANKL and promoting CFU-GM formation and osteoclastogenesis.

J. Clin. Invest. 106:887-895 (2000).

\section{Introduction}

Alcohol (ethanol) abuse is a widespread clinical disorder that is accompanied by osteoporosis and increased incidence of fractures $(1,2)$. Up to $50 \%$ of ambulatory chronic alcoholics demonstrate radiographic evidence of extensive bone loss (3). Additionally, a negative correlation between ethanol intake and axial bone mineral density (BMD) in premenopausal (4) and postmenopausal $(5,6)$ women has been demonstrated.

The mechanisms through which ethanol promotes bone loss are currently not well understood. However, there is evidence that ethanol promotes osteoporosis through alteration of both the production and resorption arms of bone remodeling. For example, ethanol inhibits proliferation of human osteoblasts (7) and chick calvarial cells (8) in vitro. Additionally, ethanol increases bone resorption in chick tibiae (8) and rat trabecular bone (9). These latter observations suggest that an osteoclastic phase of bone loss contributes to ethanol-mediated osteoporosis.

IL-6, a multifunctional cytokine produced by a great diversity of cells, is recognized as an important regulator of the immune and hematopoietic systems (reviewed in ref. 10). IL-6 has joined ranks with other cytokines/growth factors such as IL- $1 \alpha$, IL- $1 \beta$, and TNF- $\alpha$ as being an important contributor to the process of bone resorption (reviewed in ref. 11). Although several studies could not document an influence of IL- 6 on bone (12-15), the bulk of research supports IL-6's role in bone resorption (reviewed in ref. 16).

On the basis of the reports that demonstrate IL-6's ability to induce osteoclastogenesis and bone loss, and the observation that ethanol induces IL- 6 gene expression in a bone marrow stromal cell line (17), we have hypothesized that ethanol induces bone loss through its ability to induce IL-6-mediated osteoclastogenesis. To test this hypothesis, we have performed in vitro and in vivo studies to determine whether IL-6-deficient mice $\left(i l 6^{--}\right)$are protected from ethanol-induced bone loss. In addition, we have investigated whether ethanolinduced bone loss is linked to osteoclastogenesis.

\footnotetext{
Methods

Animals. Male 8-week-old C57/BL6 mice $\left(\mathrm{il6}^{+/+}\right)$or $\mathrm{il6}^{-/-}$ backcrossed on a C57/BL6 background (18) for eight generations were individually housed in plastic cages
} 
under standard laboratory animal conditions. Mice were fed the National Research Council (NRC) liquid diet (19), which contains $100 \%$ of the vitamin and mineral requirements set by the NRC. The ethanol-treated group diet contained $26 \%$ of energy as ethanol, $31.5 \%$ as carbohydrates, $12.5 \%$ as protein, and $30 \%$ as fat (diet no. 710279; Dyets, Bethlehem, Pennsylvania, USA) as described previously (20), resulting in a dietary ethanol concentration of $5 \%$ (vol/vol) and blood ethanol concentrations of 9.9 and $7.3 \mathrm{mmol} / \mathrm{l}$ at 5 and 20 hours after feeding, respectively (19). An isocaloric level of maltose was substituted for ethanol in the nonethanol control diet. Diets were prepared fresh daily. To minimize differences due to food intake, mice were pair fed. To accomplish this, food intake for each ethanol-fed mice was measured, then its respective pair-fed control diet-fed mouse received the same volume of food the following day. Animals were fed for 4 months and were then sacrificed by $\mathrm{CO}_{2}$ asphyxiation. Liver was collected and stored in formalin for standard histopathological evaluation. All outcome measurements were performed on individual mouse samples, i.e., there was no pooling of samples. The University of Michigan Animal Care and Use Committee approved the animal protocols.

Bone densitometry. BMD was measured using dualenergy x-ray absorptiometry (DEXA) on an Eclipse peripheral Dexa Scanner using pDEXA Sabre software, version 3.9.4 in research mode (Norland Medical Systems, Fort Atkinson, Wisconsin, USA). To measure whole-body BMD, mice were anesthetized with ketamine before and after ethanol feeding and were placed in sternal recumbency on the scanner. The mice were scanned at $10 \mathrm{~mm} / \mathrm{s}$ with a resolution of $0.5 \mathrm{~mm} \times 0.5$ $\mathrm{mm}$. BMD was determined in a window that excluded the calvarium and tail. To measure femoral BMD, the right femur was excised from soft tissue and placed on the scanner in lateral position. The femur was scanned at $5 \mathrm{~mm} / \mathrm{s}$ with a resolution of $0.1 \mathrm{~mm} \times 0.1 \mathrm{~mm}$. BMD was determined in a window that encompassed the entire femur. Short-term BMD precision (percent coefficient of variation) was approximately $3 \%$ for both of these techniques.

Quantification of deoxypyridinolines. At the time of sacrifice, urine was collected and frozen at $-80^{\circ} \mathrm{C}$ until assayed. Urine creatinine was measured using a creatinine kit (Metra Biosystems, Mountain View, California, USA) as recommended by the manufacturer. Deoxypyridinolines (Dpd's) were measured using the Pyrilinks-D kit (Metra Biosystems) as recommended by the manufacturer. A standard curve was created using a four-parameter fit generated by MetraFIT software (Metra Biosystems). Dpd was corrected for variations in urine concentration by dividing the Dpd value ( $\mathrm{nmol} / \mathrm{l}$ ) by the creatinine value ( $\mathrm{mmol} / \mathrm{l}$ ) for each sample.

Quantification of IL-6, estradiol, testosterone, and ethanol. Serum samples were collected before treatment using retro-orbital venipuncture, and, after treatment, using cardiac puncture. Samples were measured immediately for ethanol levels and frozen at $-80^{\circ} \mathrm{C}$ until assayed for IL-6, estrogen, and testosterone. IL-6 was measured using a murine ELISA kit (Murine IL-6 Quantikine kit; R\&D Systems, Minneapolis, Minnesota, USA) as recommended by the manufacturer. Ethanol levels were determined by the measuring the ability of alcohol dehydrogenase to form acetaldehyde from the blood sample as recommended by the manufacturer (Alcohol Level Kit; Sigma Chemical Co., St. Louis, Missouri, USA). Estradiol levels were measured using ELISA (17 $\beta$-Estradiol ELISA; KMI Diagnostics, Minneapolis, Minnesota, USA) as recommended by the manufacturer. Total testosterone levels were measured by RIA (Testosterone RIA kit; Diagnostic Systems Laboratories Inc., Webster, Texas, USA).

Quantification of CFU-fibroblast and CFU-osteoblast and culture calcium retention. CFU-fibroblasts (CFU-Fs) and CFU-osteoblasts (CFU-OBs) were measured as reported previously (21), with minor modification. Briefly, mouse femora were flushed with $3 \mathrm{ml}$ of phenol red-free $\alpha$-MEM to obtain marrow cells. The cells were rinsed twice, total cell numbers per femur determined and resuspended to obtain a single-cell suspension of $1 \times 10^{7}$ cells $/ \mathrm{ml}$. For determination of CFU-Fs, $1.5 \times 10^{6}(150$ $\mu \mathrm{l})$ cells were seeded into $4 \mathrm{ml}$ of complete media $(\alpha-$ MEM, 15\% FBS, $50 \mu \mathrm{g} / \mathrm{ml}$ ascorbic acid, $10 \mathrm{mM} \beta$-glycerophosphate) $+10 \mathrm{nM}$ dexamethasone per well of sixwell plate. Assays were performed in duplicate for each animal. One half of the media was removed and replaced on day 5 with $\alpha$-MEM, containing 30\% FBS, $100 \mu \mathrm{g} / \mathrm{ml}$ ascorbic acid, $20 \mathrm{mM} \beta$-glycerophosphate to result in a final concentration of the original complete media. Dexamethasone was not added after the initial culture period. On day 10 of culture, cells were fixed stained for alkaline phosphatase (ALP) and counter stained with hematoxylin. ALP-positive colonies containing $\geq 20$ cells were enumerated. To determine CFU$\mathrm{OB}$, cultures were established in duplicate and maintained as for CFU-Fs except $2.5 \times 10^{6}$ cells $(250 \mu \mathrm{l}$ of $1 \times$ $10^{7}$ cells $/ \mathrm{ml}$ ) were plated per well. Media were replaced every 5 days as already described here. Cells were cultured for 25 day at which time they were fixed in $18 \%$ formaldehyde, $50 \%$ ethanol, 32\% deionized water for 1 minute, and then stained using Von Kossa's method. Stained colonies were enumerated. To measure calcium content in cultures, cells were prepared and grown as for CFU-OB; then calcium content was measured using standard biochemistry (Calcium Kit, Fisher Scientific Co., Pittsburgh, Pennsylvania, USA).

Quantification of osteoclast formation in bone marrow cultures. Osteoclast formation was determined as reported previously (21), with minor modifications. Briefly, marrow cells were collected as already described here. To determine osteoclast number, the cells were plated at 1.5 $\times 10^{6}$ cells per $2 \mathrm{~cm}^{2}$ well on a $13 \mathrm{~mm}^{2}$ round tissue culture cover slips (four to six replicate cultures per mouse). The cultures were maintained for 9 days in the presence of $10^{-8} \mathrm{M} \mathrm{1,25}(\mathrm{OH})_{2} \mathrm{D}_{3}$ with replacement of half the medium every 3 days. To confirm the identification of osteoclasts, several replicates were evaluated for tartrate- 
resistant acid phosphatase (TRAP) (Acid Phosphatase Kit; Sigma Chemical Co.) and calcitonin receptors (CTRs) by a method described previously (22) using $\left.{ }^{[125} \mathrm{I}\right]$ salmon calcitonin. Results are reported as number of multinucleated (more than three nuclei) TRAP-positive cells and CTR-positive cells per cover slip.

Assessment of individual CFU-F colonies for ability to support osteoclast formation. CFU-F osteoclastogenic activity was determined as reported previously (21), with minor modifications. Briefly, $100 \mu \mathrm{l}$ of $2 \times 10^{5}$ bone marrow cells $/ \mathrm{ml}$ in $\alpha$-MEM was placed into wells of 96-well plates containing $100 \mu \mathrm{l}$ of $\alpha$-MEM, 30\% FBS, 100 $\mu \mathrm{g} / \mathrm{ml}$ ascorbic acid, and $20 \mathrm{mM} \beta$-glycerophosphate in each well of 96-well plate. The bone marrow cells were incubated for 5 days, at which time spleen cells (for a source of osteoclastogenic precursors) were isolated from $i l 6^{+/+}$mice and resuspended to $1 \times 10^{6} / \mathrm{ml}$ in $\alpha$ MEM with 20\% FBS and $20 \mathrm{nM}$ vitamin D3. Then 100 $\mu \mathrm{l}$ of media was removed from each well, and $100 \mu \mathrm{l}$ of the spleen cell suspension was added. On day 9, one half of the media was removed and replaced with $\alpha$ MEM with $20 \%$ FBS and $20 \mathrm{nM}$ VitD3. On day 13 , the cells were stained for TRAP, and each well was examined microscopically for the presence of CFU-Fs (>20 cells) and osteoclastic cells (TRAP-positive).

Quantification of CFU-GMs. To determine CFU-GM formation, $1.25 \times 105$ of the bone marrow cells (i.e., $250 \mu \mathrm{l}$ of $5 \times 105$ cells $/ \mathrm{ml}$ ) were seeded into $2.5 \mathrm{ml}$ of a methylcellulose-based media containing CFU-GM-promoting growth factors (MethoCult HCC-3534; Stem Cell Technologies, Vancouver, British Columbia, Canada) in a $15-\mathrm{ml}$ sterile test tube. The cells were vortexed and bubbles allowed to settle. Then $1.1 \mathrm{ml}$ cell per media mixture was placed into a $35-\mathrm{mm}$ cell culture dish using a 16 gauge 1.5 -inch needle. Assays were performed in duplicate. The duplicate $35-\mathrm{mm}$ dishes with cells were placed into a $150-\mathrm{mm}$ plate along with a covered 35 $\mathrm{mm}$ dish filled with $3 \mathrm{ml}$ of sterile water. The cells were incubated at $37^{\circ} \mathrm{C} 5 \% \mathrm{CO} 2$ for 7 days, at which time CFU-GMs (defined as $>50$ cells) were counted.

Evaluation of receptor activator of NF- $\kappa B$ ligand $m R N A$ levels. Bone marrow cells were frozen at $-80^{\circ} \mathrm{C}$ until total RNA was collected using Trizol reagent (Life Technologies, Grand Island, New York, USA) as recommended by the manufacturer. PCR primers used for the detection of receptor activator of NF- $\mathrm{KB}$ ligand (RANKL), based on published sequence (23), consisted of upper primer: 5'CCATCGGGTTCCCATAAAGTCAC3' and lower primer: 5'AAAGCCCCAAAGTACGTCGCATCT3', resulting in a $\mathrm{PCR}$ product of $407 \mathrm{bp}$. Murine $\beta$-actin mRNA expression was used as an internal control. The $\beta$-actin primers consisted of upper primer: 5'GTGGGCCGCTCTAGGCACCAA3'; and lower primer: $5^{\prime}$ 'CTCTTTGATGTCACGCACGATTTC 3 ', resulting in a PCR product of $540 \mathrm{bp}$. RT-PCR was performed with 1 $\mu \mathrm{g}$ of total RNA using the Access RT-PCR system (Promega Corp., Madison, Wisconsin, USA), as directed by the manufacturer, in a thermal cycler (GeneAmp PCR system 9700; Perkin-Elmer Applied Biosystems,
Norwalk, Connecticut, USA) under the following conditions: first strand cDNA was synthesized at $48^{\circ} \mathrm{C}$ for 45 minutes and then denaturated at $94^{\circ} \mathrm{C}$ for $2 \mathrm{~min}$ utes for the first cycle and at 15 seconds for additional cycles; annealing was performed at $55^{\circ} \mathrm{C}$ for 30 seconds and extension at $72^{\circ} \mathrm{C}$ for 60 seconds. Final extension was at $72^{\circ} \mathrm{C}$ for 5 minutes. To enable semiquantitation, 30 cycles of amplification were performed, which was in the log-linear range of amplification for RANKL. The PCR products were subjected to electrophoresis on a $1.5 \%$ agarose gel, stained with ethidium bromide, and band density measured by densitometry. The density of the RANKL PCR product band was divided by the density of the $\beta$-actin PCR product band for each sample.

Bone histomorphometry. The right femur was dissected from soft tissue, fixed in 10\% phosphate-buffered formalin for 24 hours, decalcified in 14\% EDTA, and embedded in a lateral position in paraffin. Longitudinal sections $(5-\mu \mathrm{M}$ thick) from the middle of the femur were stained with hematoxylin and eosin or trichrome stain. Histomorphometry was performed using Bioquant Nova Imaging Software (Bioquant, Nashville, Tennessee, USA) and digitizer tablet (Summagraphics Corp., Seymour, Connecticut, USA). Histomorphometric parameters of the secondary spongiosa in the distal femoral metaphysis were measured in standard rectangular area $\left(1.5 \mathrm{~mm}^{2}\right)$ at least $0.2 \mathrm{~mm}$ proximal to the distal femoral growth plate, to exclude primary spongiosa (24). Parameters were measured at a magnification of 100 . The terminology used was that recommended by the Histomorphometry Nomenclature Committee of the American Society for Bone and Mineral Research (25).

Statistics. Repeated measures ANCOVA was used to test for significant differences in mean BMD among groups, using weight as a covariate. Repeated measure ANOVA was used to test for significant differences between pre- and post-treatment IL-6 levels. Factorial ANOVA was used to test for significant differences in parameters, other than BMD or IL-6, among groups. Fisher's least significant difference was used for post hoc analysis. Statistical significance was determined as $P=0.05$.

\section{Results}

$\mathrm{Il6}^{+/+}$and $i l 6^{-/-}$6-month-old male mice on a C57/B16 background were pair fed 5\% ethanol or maltose-control liquid diets for 4 months. Blood ethanol levels, 4 hours after an individual feeding, were $9.6 \pm 2.5 \mathrm{mmol} / \mathrm{l}$ in ethanol-fed mice compared to $0.23 \pm 1.2 \mathrm{mmol} / \mathrm{l}$ in control-fed mice $(P<0.001)$ at 4 months after initiation of diet. The levels present in the ethanol-fed mice represent the blood levels obtained in chronic alcoholic patients $(26,27)$.

At the end of the study period, mice were sacrificed and evaluated for a variety of parameters. To rule out hepatic inflammation or cirrhosis, common sequelae to chronic ethanol ingestion, the liver was evaluated for pathological changes. There was no evidence 
$\mathbf{a}$

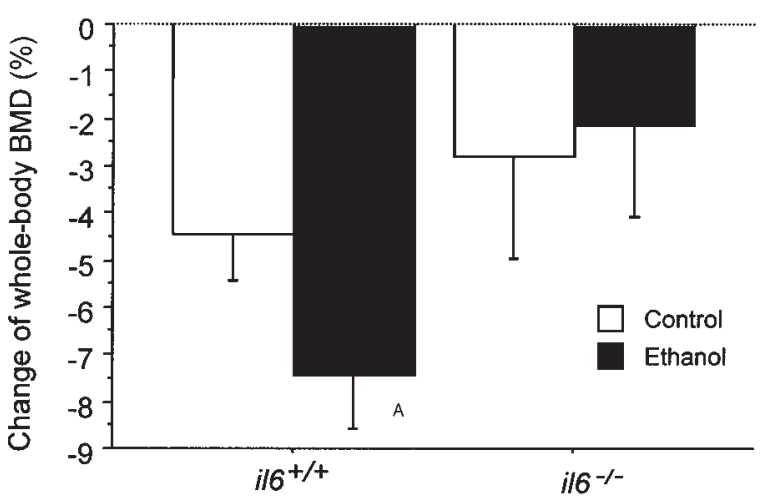

b

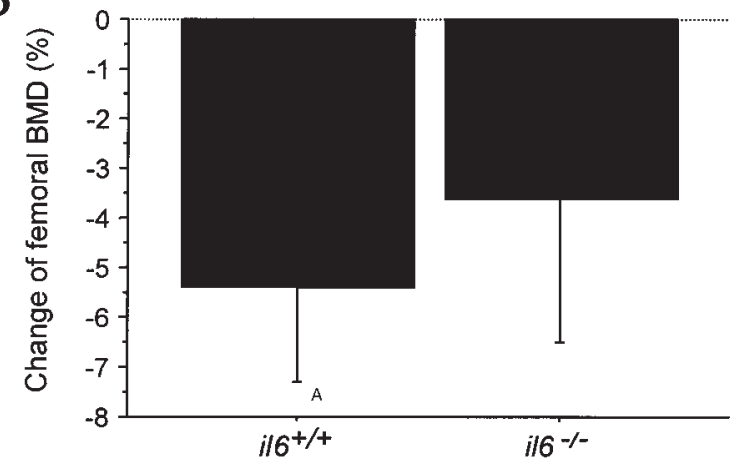

Figure 1

Effect of ethanol ingestion on whole-body and excised femoral BMD in wild-type and IL- 6 gene knockout mice. $1 / 6^{+/+}$and il $6^{-/-}$mice were fed either a control diet or $5 \%$ ethanol diet for 4 months. (a) Wholebody BMD was measured before and 4 months after initiation of the diet. (b) Femoral BMD was measured from excised left femurs 4 months after initiation of diet. Data are reported as (a) mean $( \pm \mathrm{SD})$ percent change in whole-body BMD measured at 4 months relative to the prediet $\mathrm{BMD}$ or $(\mathbf{b})$ mean $( \pm \mathrm{SD})$ percent difference between femoral BMD in ethanol-fed mice compared with pair-fed matched control-fed mice. Data were analyzed using ANCOVA using bodyweight as a covariate and Fisher's least significant difference for post hoc analysis. ${ }^{A} P<0.03$ vs. control-fed $i / 6^{+/+}$mice. Measurements were performed in eight to ten individual mice per group.

of gross or histological liver pathology in any animal. Ethanol has been reported to modulate gonadal hormone levels $(28,29)$, which in turn can modify bone remodeling. Therefore, we evaluated serum estradiol and testosterone levels. Both estradiol and testosterone levels were similar among all groups of animals (ANOVA; data not shown). To confirm that ethanol induced IL-6 expression in vivo, we measured serum IL- 6 levels before initiation of ethanol feeding and at the end of the study. Serum IL- 6 levels were nondetectable (ELISA sensitivity $=3 \mathrm{pg} / \mathrm{ml}$ ) in all the $i l 6^{+/+}$mice before the initiation of ethanol feeding. At the end of the study, serum IL-6 levels were below the ELISA nondetectable in the controlfed $i l 6^{+/+}$mice; whereas they were $33.6 \pm 5.3 \mathrm{pg} / \mathrm{ml}$ in the ethanol-fed $i l 6^{+/+}$mice. For statistical analysis, we substituted the minimal detectable level of $3 \mathrm{pg} / \mathrm{ml}$ (which is more conservative than placing $0 \mathrm{pg} / \mathrm{ml}$ ) for mice with undetectable IL-6 levels. There was a significant increase $(P<0.001$, repeated measures ANOVA) in IL-6 levels in the ethanol-fed mice. Taken together, these data demonstrate that ethanol ingestion induces IL- 6 expression in the absence of liver pathology and independent of modulating estradiol or testosterone levels.

To determine whether ethanol induces bone loss in mice, BMD was measured using DEXA. Whole-body BMD before and after ethanol treatment, and postethanol treatment BMD of the excised right femur were quantified. The whole-body BMD decreased by $3.01 \pm 1.0 \%$ in the ethanol-fed $i l 6^{+/+}$mice compared with the control-fed $i l 6^{+/+}$mice (Figure 1a). However, there was no decrease in the ethanol-fed $i l 6^{-/-}$mice compared with the control-fed $i l 6^{-/-}$mice (Figure 1a). Consistent with these findings, femoral BMD decreased by $5.37 \pm 1.93 \%$ in the ethanol-fed $i l 6^{+/+}$ mice compared with the control-fed $i l 6^{+/+}$mice (Figure $1 b)$. In contrast, there was no significant decrease of $\mathrm{BMD}$ in the ethanol-fed $i l 6^{-/-}$mice compared with the control-fed $i l 6^{-/}$mice (Figure $\left.1 b\right)$. To support the BMD findings further, urinary Dpd level, an indicator of bone resorption, was quantified. In agreement with the BMD data, Dpd levels were mildly elevated in the ethanol-fed compared with the control-fed $i l 6^{+/+}$ mice, but the Dpd levels were similar in the ethanolfed and control-fed $i l 6^{-/-}$mice (Figure 2). These data establish that IL-6 is required for ethanol to induce bone loss. Furthermore, the increased Dpd levels suggest that increased bone resorption is a component of the IL-6-mediated activity.

IL-6 induces osteoclastogenesis (30); thus, if ethanol requires IL- 6 to induce bone loss, ethanol may promote bone loss through induction of osteoclastogenesis. To

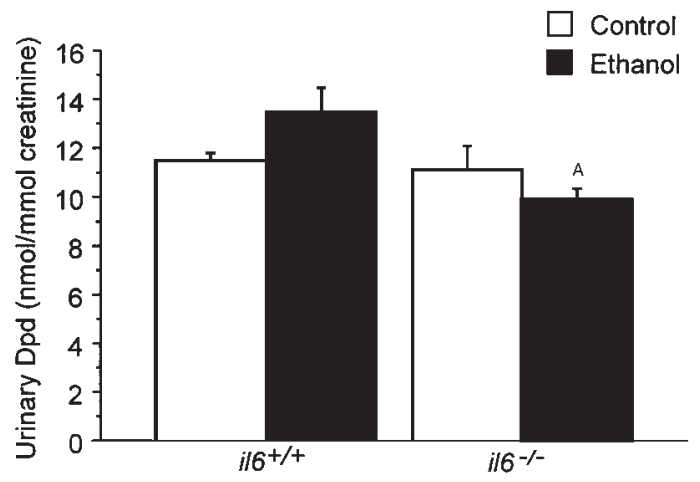

Figure 2

Effect of ethanol ingestion on urinary deoxypyridinolines in wildtype or IL- 6 gene knockout mice. $1 / 6^{+/+}$or $i l 6^{-/-}$mice were fed either a control diet or $5 \%$ ethanol diet for 4 months. The mice were then sacrificed, and urine was collected and evaluated for deoxypyridinolines (Dpd's) and creatinine levels as described in Methods. Data are presented as mean $( \pm S D)$ urinary Dpd corrected for creatinine levels. Data were analyzed using ANOVA and Fisher's least significant difference for post hoc analysis. ${ }^{A} P=0.045$ compared with control-fed $i 6^{+/+}$mice. Measurements were performed in six to eight individual mice per group. 
evaluate this hypothesis, we determined the effect of ethanol ingestion on formation of bone marrow CFUGMs, which are the osteoclast precursors. CFU-GMs were increased approximately $40 \%$ in ethanol-fed $\mathrm{il6}^{+/+}$ animals (Figure 3a). In contrast, ethanol ingestion did not increase CFU-GMs in il6 $6^{-/}$mice (Figure 3a). In parallel studies, the effect of ethanol ingestion and the requirement of IL- 6 on formation of osteoclast-like cells was examined in bone marrow cell cultures. Similar to findings for CFU-GMs, ethanol-fed $i 6^{+/+}$mice exhibited approximately a $45 \%$ increase of osteoclastlike cells (Figure 3b). This increase was not observed in bone marrow from the $i 6^{-/}$mice (Figure $3 \mathrm{~b}$ ). To ensure the increases of CFU-GMs and osteoclast-like cells were not due to a general increase in bone marrow cell number, we determined the number of cells obtained per femur. There was an average of $2.2 \pm 0.73 \times 10^{7}$ cells per femur, and no differences were detected among the groups (data not shown). Taken together, these data suggest that ethanol induces CFU-GM formation, resulting in osteoclastogenesis through IL-6. In turn, IL-6 is known to induce RANKL expression $(31,32)$, which induces osteoclastogenesis through binding to RANK on the cell membrane of osteoclastogenic precursors $(33,34)$. Thus, it follows that ethanol may also promote osteoclastogenesis through IL-6-induced RANKL expression. Accordingly, we semiquantitated RANKL mRNA expression in the bone marrow of the mice. Ethanol ingestion induced approximately a 70\% increase of RANKL mRNA expression in the $i 6^{+/+}$animals (Figure 3c). In contrast, ethanol ingestion did not increase RANKL mRNA expression in il6 $6^{-/-}$mice (Figure 3c). These data are consistent with IL-6's ability to induce RANKL expression and osteoclastogenesis.

To assess further the effect of ethanol on the capability of the bone marrow microenvironment to support osteoclastogenesis, we determined the ability of CFUFs from il6 $6^{+/+}$and $i l 6^{-/-}$ethanol-fed or control-diet-fed mice to induce osteoclast-like cells in single-cell suspensions of spleen cells from $i 6^{+/+}$mice (which serve as
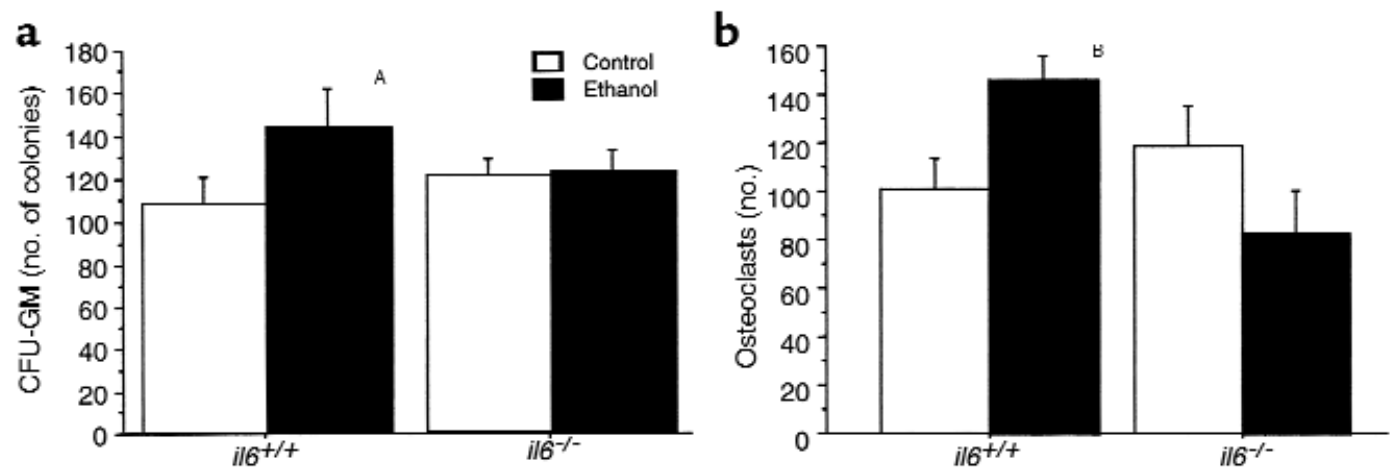

C
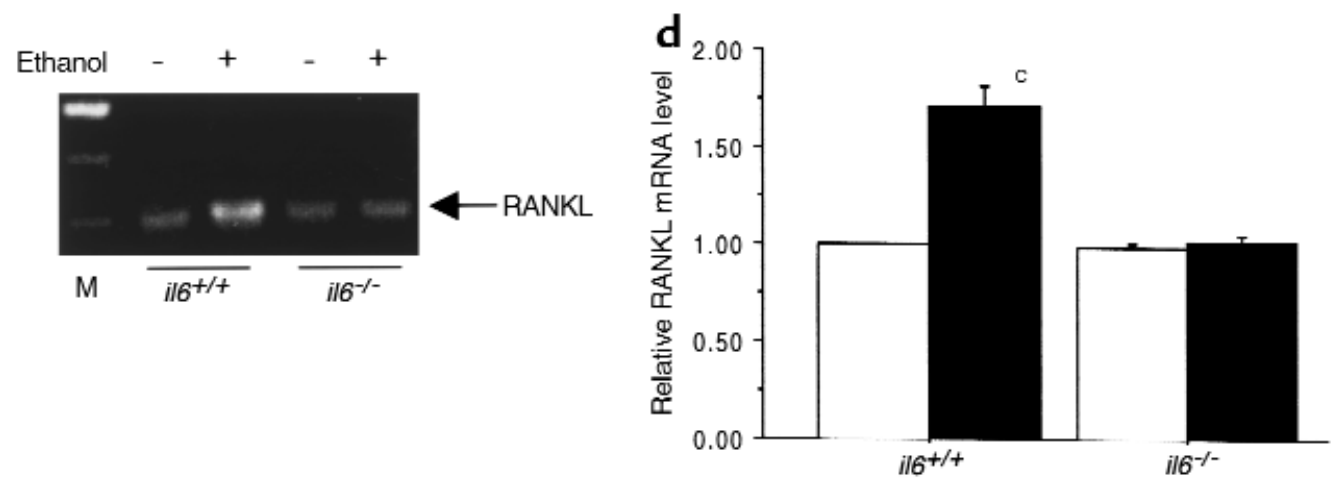

\section{Figure 3}

Effect of ethanol ingestion on CFU-GM formation, osteoclastogenesis, and RANKL mRNA expression in the bone marrow of wild-type or IL-6 gene knockout mice. $1 / 6^{+/+}$or $i 6^{-/-}$mice were fed either a control diet or $5 \%$ ethanol diet for 4 months. The mice were then sacrificed, and bone marrow was collected from the femur. (a) CFU-GM formation was determined as described in Methods. Data are presented as mean $( \pm \mathrm{SD})$ CFU-GM formed per $5.5 \times 10^{5}$ nucleated bone marrow cells. (b) Osteoclast formation was determined in mar-

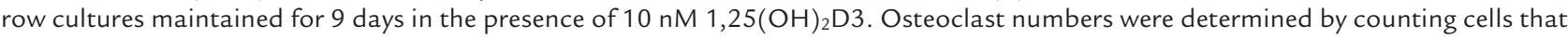
bound I-125-calcitonin and were TRAPase-positive as described in Methods. Data are presented as mean ( \pm SD) osteoclasts per $1.5 \times$ $10^{6}$ nucleated bone marrow cells. (c) RANKL mRNA expression was determined by subjecting bone marrow cell total RNA to RT-PCR for RANKL and $\beta$-actin mRNA as described in Methods. Density of individual bands was determined. A representative gel is shown. Data are presented as mean $( \pm S D)$ relative density of RANKL PCR product bands normalized with $\beta$-actin PCR product bands. (d)Data were analyzed using ANOVA and Fisher's least significant difference for post hoc analysis. ${ }^{A} P<0.001$ compared with control-fed $i / 6^{+/+}$mice. ${ }^{B} P=$ 0.033 compared with control-fed $i / 6^{+/+}$mice. ${ }^{C} P<0.01$ compared with control-fed il $6^{+/+}$mice. Measurements were performed on ten individual mice per group for CFU-GM and osteoclast studies and four individual mice per group for RANKL mRNA determination. 


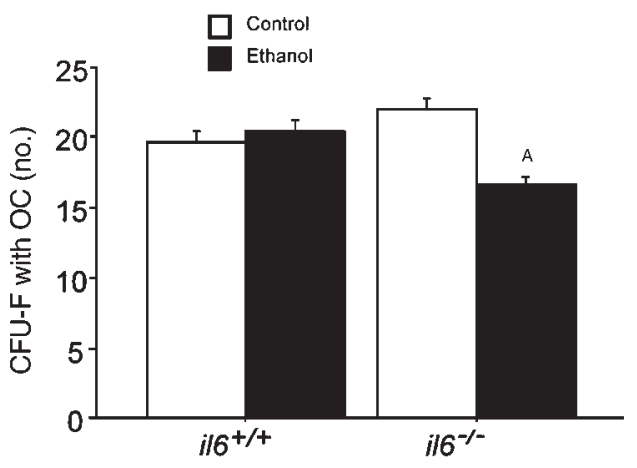

Figure 4

Effect of ethanol ingestion of the ability of CFU-F to support osteoclastogenesis. $116^{+/+}$or $i 16^{-/-}$mice were fed either a control diet or $5 \%$ ethanol diet for 4 months. The mice were then killed, and bone marrow was collected from the femur. CFU-F cultures, from individual animals, were established in 96-well plates for 5-days; then singlecell suspension from spleens of $i / 6^{+/+}$mice were added to the wells. The cultures were maintained for an additional 8 days and then stained for TRAP. Each well was then examined microscopically for the presence of CFU-F ( $>20$ cells) and osteoclast-like cells (TRAPpositive). Data are presented as mean ( \pm SD) CFU-F with osteoclasts. Data were analyzed using ANOVA and Fisher's least significant difference for post hoc analysis. ${ }^{A} P<0.01$ compared with control-fed il $6^{-/-}$mice. Measurements were performed on ten individual mice per group. OC, osteoclasts.

a source of normal osteoclast precursors). Specifically, bone marrow cells isolated from $i 6^{+/+}$and $i l 6^{-/-}$mice were allowed to develop into CFU-Fs for 5 days, then overlayed with splenic cells from normally fed $i l 6^{+/+}$ mice as a source of osteoclast precursors. After an additional 8 days, the number of CFU-Fs containing TRAP+ osteoclast-like cells was quantified. Ethanol ingestion by $i l 6^{+/+}$mice did not affect the number of CFU-Fs that produced osteoclast-like cells compared to control diet-fed $i l 6^{+/+}$mice (Figure 4). In contrast, ethanol ingestion in $i l 6^{-/-}$mice reduced the number of CFU-F with osteoclast-like cells compared with the control diet-fed $i l 6^{-/}$mice (Figure 4). These results suggest that (a) ethanol does not increase osteoclastogenesis through CFU-Fs, and (b) IL-6 protects CFU-Fs from a toxic effect of ethanol.

To assess for the presence of diminished bone osteoblast function secondary to ethanol ingestion, we measured CFU-F formation, CFU-OB formation, and cell culture calcium retention in long-term marrow cultures containing $\beta$-glycerophosphate and ascorbic acid. Ethanol ingestion had no effect on CFU$\mathrm{F}$ formation (Figure 5a). Ethanol ingestion diminished the number of CFUOBs and the cell culture calcium content by approximately $15 \%$ and $20 \%$, respectively, compared to $i l 6^{+/+}$mice fed control diet (Figure 5, b and c). In contrast, there was no alteration of mineralized

\section{Table 1}

colony number in $i l 6^{-/-}$mice (Figure $5, \mathrm{~b}$ and c). However, ethanol ingestion decreased cell culture calcium content by approximately $60 \%$, compared with the control-fed $i l 6^{-/-}$mice.

To determine whether these in vitro observations were reflected in vivo, bone histomorphometry was performed on the femur. There was no difference of bone histomorphometric parameters between controlfed $i l 6^{+/+}$and $i l 6^{-/-}$mice (Table 1). However, the cancellous bone volume was decreased in the ethanol-fed versus control-fed $i l 6^{+/+}$mice, but not the ethanol-fed versus control-fed $i l 6^{-/-}$mice, which demonstrates that ethanol induces bone loss through IL-6. Additionally, the osteoclast perimeter was increased in the ethanolfed $i l 6^{+/+}$mice femurs but not the ethanol-fed il6-/mice, which suggests that (a) increased osteoclast activity contributes to ethanol-induced bone loss, consistent with the ability of ethanol to induce CFU-GMs and osteoclastogenesis in vitro, and (b) ethanol requires IL-6 to mediate this activity. Finally, the osteoblast perimeter was decreased in both the ethanolfed $i l 6^{+/+}$and the ethanol-fed $i l 6^{-/-}$mice femurs, suggesting that decreased osteoblast-produced bone contributed to ethanol-mediated bone loss and was consistent with the ability of ethanol to inhibit CFU$\mathrm{OB}$ production in vitro.

\section{Discussion}

Although the ability of ethanol to induce osteopenia has been recognized for decades, the mechanism through which this occurs has not been elucidated. The results from the present study demonstrate that chronic ethanol ingestion increases osteoclastogenesis and bone loss through IL-6 in mice. These findings are consistent with the observation that ethanol induces bone resorption in chick tibiae (8) and rat trabecular bone (9). Furthermore, these findings are in line with the evidence that IL-6 mediates osteoclastogenesis and bone loss in estrogen- and androgen-deficient states $(30,35)$. Additionally, they clarify the cellular changes underlying the increased bone resorption associated with ethanol ingestion. Taken together with our current observation that ethanol induces IL-6 expression in vivo and earlier observa-

Static histomorphometry of distal femoral cancellous bone in $i / 6^{+/+}$and $i l 6^{-/-}$mice fed a control or ethanol liquid diet for 4 months

\begin{tabular}{lcccc}
\hline & \multicolumn{2}{c}{$i l 6^{+/+}$} & \multicolumn{2}{c}{$i l 6^{-/-}$} \\
& Control & Ethanol & Control & Ethanol \\
Cancellous bone area $(\%)$ & $28.7 \pm 0.9$ & $22.6 \pm 0.6^{\mathrm{A}}$ & $27.0 \pm 0.4$ & $25.6 \pm 0.6$ \\
Osteoclast perimeter $(\%)$ & $2.3 \pm 0.2$ & $3.6 \pm 0.3^{\mathrm{A}}$ & $2.1 \pm 0.4$ & $2.7 \pm 0.2$ \\
Osteoblast perimeter $(\%)$ & $3.6 \pm 0.5$ & $2.6 \pm 0.6^{\mathrm{A}}$ & $3.7 \pm 0.5$ & $2.7 \pm 0.7^{\mathrm{B}}$ \\
Trabecular number $\left(\mathrm{mm}^{-1}\right)$ & $3.0 \pm 0.5$ & $3.2 \pm 0.5$ & $3.8 \pm 0.5$ & $3.7 \pm 0.7$ \\
Trabecular width $(\mu \mathrm{M})$ & $48.6 \pm 1.8$ & $39.4 \pm 0.7^{\mathrm{A}}$ & $42.4 \pm 0.7$ & $42.7 \pm 0.8$ \\
Trabecular spacing $(\mu \mathrm{M})$ & $439.0 \pm 13.3$ & $363 \pm 16.7^{\mathrm{A}}$ & $441.3 \pm 12.3$ & $425.2 \pm 19.7$
\end{tabular}

Data are presented as mean \pm SEM. $n=6$ mice per group. ${ }^{A} P<0.01$ versus $i / 6^{+/+}$control-diet-fed mice. ${ }^{\mathrm{B}} P<0.01$ versus il $6^{-/-}$control-diet-fed mice. 
tions that IL- 6 induces bone loss through osteoclastogenesis (30), it appears that IL-6 mediates the effects of ethanol on bone loss in this murine model.

The role of bone resorption in ethanol-induced bone loss has not previously been well documented. Taken together, our data demonstrate that ethanol induces bone resorption. The modest increase of urinary Dpd levels induced by ethanol is only suggestive that ethanol induces bone resorption. This observation is consistent with the finding of increased urinary excretion of hydroxyproline in chronic alcoholic patients (36). Stronger evidence that ethanol induces osteoclastogenic activity was provided by the observation of increased osteoclastogenesis in bone marrow cell cultures and the bone histomorphometry finding of increased osteoclast perimeter in the ethanol-fed $i 6^{+/+}$ mice. These results are consistent with histomorphometric measurements by Diez et al. (37) and Johnell et al. (38) who observed increased resorptive surface and osteoclast number in alcoholic men. However, our data contrast with those of Crilly et al. (39), who did not observe a change in osteoclastic parameters in alcoholic men. Differences in patient populations (e.g., age and duration of ethanol abuse) are likely to account for the discrepancies between these earlier reports. Our data cannot conclusively delineate whether increased bone resorption or decreased bone production is the major mediator of ethanol-induced bone loss, because both increased resorptive parameters and decreased osteoblast function were observed in the ethanol-fed mice.

Hepatitis and hepatic cirrhosis are common sequelae of chronic ethanol abuse. This nidus of inflammation may lead to general overexpression of inflammatory cytokines and malnutrition that promote bone resorption. To minimize confounding from hepatic disease, we fed the mice for a period that would not induce cirrhosis. The absence of hepatic inflammation and cirrhosis was confirmed by histology; however, bone loss was observed. This observation is consistent with pre-

\section{Figure 5}

Effect of ethanol ingestion on osteoblast parameters in wild-type or IL6 gene knockout mice. $116^{+/+}$or $i 16^{-/-}$mice were fed either a control diet or $5 \%$ ethanol diet for 4 months. The mice were then killed and bone marrow was collected from the femur. CFU-F, CFU-OB, and Ca. (a) To assess the marrow's ability to support osteoblastogenesis, marrow cultures were maintained for 10 days in the presence of $50 \mu \mathrm{g} / \mathrm{ml}$ ascorbic acid and $10 \mathrm{mM} \beta$-glycerophosphate. CFU-Fs were determined by counting ALP-positive colonies as described in Methods. ( $\mathbf{b}$ and $\mathbf{c}$ ) To assess for the marrow's ability to support osteoblast function, marrow cultures were maintained for 25 days in the presence of $50 \mu \mathrm{g} / \mathrm{ml}$ ascorbic acid and $10 \mathrm{mM} \beta$-glycerophosphate. (b) CFU-OBs were determined by counting von Kossa-positive colonies and (c) total calcium in the cultures was determined as described in Methods. Data are reported as mean ( \pm SD). Data were analyzed using ANOVA and Fisher's least significant difference for post hoc analysis. ${ }^{A} P<0.01$ compared with control-fed il $6^{-1-}$ mice. ${ }^{B} P=0.02$ compared with control-fed il $6^{-/-}$mice. $C P=0.005$ compared with control-fed il $6^{-/-}$mice. Measurements were performed on ten individual mice per group. vious reports in humans that demonstrate bone loss in the absence of cirrhosis $(37,40)$. These results demonstrate that ethanol induces bone loss through a mechanism other than nonspecific hepatic inflammation.

The observations that ethanol decreased (a) total BMD, (b) femoral BMD, and (c) cancellous bone area and increased (a) osteoclast perimeter and (b) osteoclast number in marrow cultures from the $i l 6^{+/+}$but not the $i l 6^{-/-}$mice unequivocally demonstrates that ethanol requires IL- 6 to induce bone loss in mice. These data are consistent with IL-6's ability to promote bone-resorption through stimulating osteoclastogenesis $(12,41-43)$. Furthermore, these results parallel those of Poli et al. (44) and Bellido et al. (35), who demonstrated that IL-6-deficient mice are protected from gonadectomy-induced osteopenia. However, results derived from $i l 6^{-/-}$mice must be interpreted with the caveat that they may have bone modeling defects and remodeling defects and thus their mature skeleton may not be directly comparable to that of the
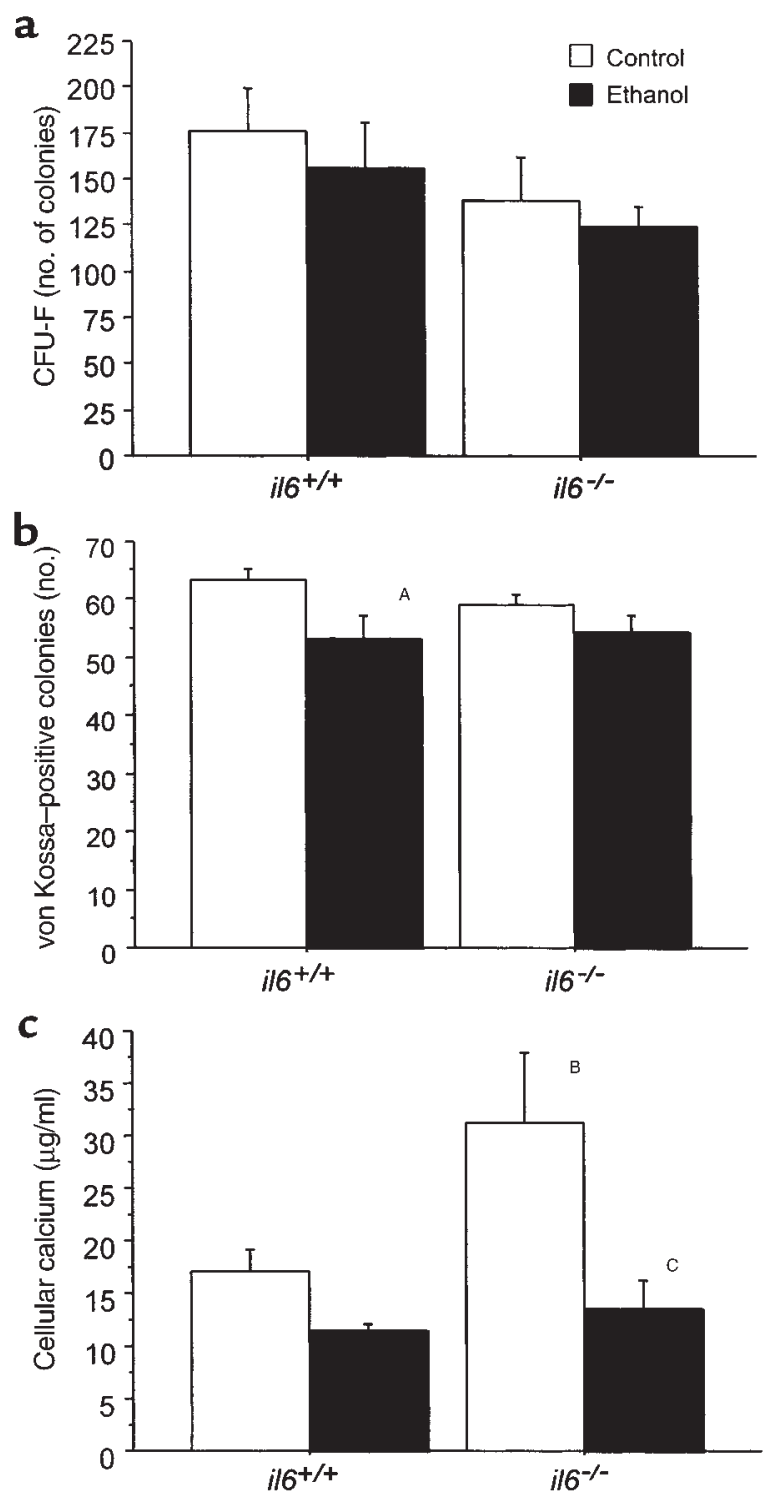
wild-type parental line. Our observation that there was no difference of histomorphometric parameters between control-fed $i l 6^{+/+}$and $i l 6^{-/-}$mice, as observed by Bellido et al. (35), suggests that such differences have a minimal impact on our results.

Ethanol-induced bone loss and increased osteoclastogenesis were associated with increased CFU-GM formation in the $i 6^{+/+}$mice. However, ethanol did not induce bone loss, osteoclastogenesis or CFU-GM in the $i l 6^{-/-}$mice. These findings, taken together with previous studies that demonstrate IL- 6 induces CFU-GMs $(30,45-47)$, suggest that ethanol promotes bone loss, in part, through IL-6-mediated induction of CFU$\mathrm{GM}$, which in turns leads to osteoclastogenesis. However, the observation that ethanol did not modify the ability of CFU-Fs to support osteoclastogenesis in the $i l 6^{+/+}$mice suggests that ethanol mediates osteoclastogenesis through some factor independent of stromal support. The observation that ethanol induced RANKL mRNA in bone marrow cells from the $i l 6^{+/+}$ mice but not the $i l 6^{-/-}$mice suggests that RANKL mediates the IL- 6 effect of ethanol on bone loss. This finding is consistent with the previous report the IL-6 stimulates RANKL production in human and murine bone marrow $(31,32)$.

In addition to increasing bone resorption, ethanolingestion reduced indices of osteoblast function. These findings are consistent with previous reports that bone histomorphometric indices of osteoblastic activity (2) and osteocalcin levels $(2,48,49)$ are decreased in chronic alcoholic patients. At the cellular level, ethanol has been shown to inhibit proliferation of rat osteosarcoma cells (50) and human osteoblast-like cells $(51,52)$ in vitro, which is consistent with our observation of decreased CFU-OB formation in the ethanol-fed $i l 6^{+/+}$mice. Additionally, ethanol and its metabolite, acetaldehyde, have been shown to inhibit CFU-F formation, the osteoblast precursors, in vitro (52). However, the ethanol-fed mice in the current study did not demonstrate decreased CFU-F formation. These findings are in agreement with reports that ethanol does not modulate osteoblastic cell number (53) or requires very high concentrations to do so (54). Differences in ethanol metabolism in vivo versus in vitro, dosage of ethanol, or duration of exposure may account for these discrepancies. Ethanol ingestion decreased the amount of calcium retained in the cultures, suggesting that it inhibits osteoblast function. In the current report, ethanol's decrease of osteoblast surface area and cell culture calcium retention was similar in both the $i l 6^{+/+}$ and $i l 6^{-/}$mice. These data suggest that ethanol represses osteoblast function independently of IL-6.

In conclusion, deletion of the IL- 6 gene protected mice from ethanol-induced bone loss. Furthermore, the absence of IL- 6 diminished the ability of ethanol to induce CFU-GM, osteoclast-like cells, RANKL mRNA, and trabecular-associated osteoclasts. Thus, there appears to be an absolute requirement for IL- 6 to induce ethanol-mediated osteoclastogenesis and bone loss. These results, together with the current evidence that ethanol induces serum IL- 6 expression in $i l 6^{+/+}$ mice and the previous observation that ethanol activates the IL- 6 gene promoter in bone marrow stromal cells (17), suggest the following mechanism for the pathophysiology of ethanol-induced osteopenia. Ethanol, independent of modulation of gonadal hormones, induces the IL- 6 promoter, either directly or indirectly, resulting in increased IL-6 expression. IL-6, in turn, promotes CFU-GM formation and RANKL expression, resulting in osteoclastogenesis and bone resorption. The relative contribution of increased osteoclastogenesis or decreased osteoblast activity to ethanol-induced bone loss remains unknown, but deserves further study.

\section{Acknowledgments}

We thank R. Murray for providing the $i l 6^{-/-}$mice. This work was supported by NIH grant R01 AG-15904.

1. Seeman, E., Melton, J., O’Fallon, M., and Riggs, B.L. 1983. Risk factors for spinal osteoporosis in men. Am. J. Med. 75:977-983.

2. Diamond, T., Stiel, D., Lunzer, M., Wilkinson, M., and Posen, S. 1989. Ethanol reduces bone formation and may cause osteoporosis. Am. J. Med. 86:282-288.

3. Spencer, H., Rubio, N., Rubio, E., Indreika, M., and Seitam, A. 1986. Chronic alcoholism: frequently overlooked cause of osteoporosis in men. Am. J. Med. 80:393-397.

4. Stevenson, J.C., Lees, B., Devenport, M., Cust, M.P., and Ganger, K.F. 1989. Determinants of bone density in normal women: risk factors for future osteoporosis. Br. J. Med. 298:924-928.

5. Hannan, M.T., et al. 2000. Risk factors for longitudinal bone loss in elderly men and women: the Framingham Osteoporosis Study. J. Bone Miner. Res. 15:710-720.

6. Hernandez-Avila, M., et al. 1991. Caffeine, moderate alcohol intake, and risk of fractures of the hip and forearm in middle-aged women. Am.J. Clin. Nutr. 54:157-163.

7. Friday, K.E., and Howard, G.A. 1991. Ethanol inhibits human bone cell proliferation and function in vitro. Metabolism. 40:562-565.

8. Farley, J.R., et al. 1985. Direct effects of ethanol on bone resorption and formation in vitro. Arch. Biochem. Biophys. 238:305-314.

9. Baran, D.T., et al. 1980. Effect of alcohol ingestion on bone and mineral metabolism in rats. Am. J. Physiol. 238:507-510.

10. Ershler, W.B., and Keller, E.T. 2000. Age-associated increased IL-6 gene expression, late life diseases and frailty. Annu. Rev. Med. 51:245-270.

11. Manolagas, S.C. 1995. Role of cytokines in bone resorption. Bone. 17(Suppl.):63S-67S.

12. Löwik, C.W.G.M., et al. 1989. Parathyroid hormone (PTH) and PTH-like protein (PLP) stimulate interleukin- 6 production by osteogenic cells: a possible role of interleukin-6 in osteoclastogenesis. Biochem. Biophys. Res. Commun. 162:1546-1552.

13. Barton, B.E., and Mayer, R. 1990. IL-3 and IL-6 do not induce bone resorption in vitro. Cytokine. 2:217-220.

14. Al-Humidan, A., et al. 1991. Interleukin-6 does not stimulate bone resorption in neonatal mouse calvariae. J. Bone Miner. Res. 6:3-8.

15. Littlewood, A.J., Aarden, L.A., Evans, D.B., Russell, R.G.G., and Gowen, M. 1991. Human osteoblastlike cells do not respond to interleukin-6.J. Bone Miner. Res. 6:141-148.

16. Jilka, R.L. 1998. Cytokines, bone remodeling, and estrogen deficiency: a 1998 update. Bone. 23:75-81.

17. Keller, E.T., Zhang, J., and Ershler, W.B. 1997. Ethanol activates the interleukin-6 promoter in a human bone marrow stromal cell line. J. Gerontol. A Biol. Sci. Med. Sci. 52:B311-B317.

18. Dalrymple, S.A., et al. 1995. Interleukin-6-deficient mice are highly susceptible to Listeria monocytogenes infection: correlation with inefficient neutrophilia. Infect. Immun. 63:2262-2268.

19. Watzl, B., et al. 1993. Diet and ethanol modulate immune responses in young C57BL/6 mice. Alcohol Clin. Exp. Res. 17:623-630.

20. Wang, Y., and Watson, R.R. 1994. Chronic ethanol consumption prior to retrovirus infection alters cytokine production by thymocytes during murine AIDS. Alcohol. 11:361-365.

21. Jilka, R.L., Weinstein, R.S., Takahashi, K., Parfitt, A.M., and Manolagas, S.C. 1996. Linkage of decreased bone mass with impaired osteoblasto- 
genesis in a murine model of accelerated senescence. J. Clin. Invest. 97:1732-1740

22. Lee, S.K., Goldring, S.R., and Lorenzo, J.A. 1995. Expression of the calcitonin receptor in bone marrow cell cultures and in bone: a specific marker for the differentiated osteoclast that is regulated by calcitonin. Endocrinology. 136:4572-4581.

23. Anderson, D.M., et al. 1997. A homologue of the TNF receptor and its ligand enhance T-cell growth and dendritic-cell function. Nature. 390:175-179.

24. Kimmel, D.B., et al. 1993. The effect of recombinant human (1-84) or synthetic human (1-34) parathyroid hormone on the skeleton of adult osteopenic ovariectomized rats. Endocrinology. 132:1577-1584.

25. Parfitt, A.M., et al. 1987. Bone histomorphometry: standardization of nomenclature, symbols, and units. Report of the ASBMR Histomorphometry Nomenclature Committee. J. Bone Miner. Res. 2:595-610.

26. Haeckel, R., and Bucklitsch, I. 1987. The comparability of ethanol concentrations in peripheral blood and saliva. J. Clin. Chem. Clin. Biochem. 25:199-204.

27. Winek, C.L., and Esposito, F.M. 1987. The validity of urine alcohol analysis in drunk drivers. In Legal medicine. C.H. Wecht, editor. Praeger Publisher. New York, New York, USA. 97-106.

28. Tadic, S.D., Elm, M.S., Subbotin, V.M., and Eagon, P.K. 2000. Hypogonadism precedes liver feminization in chronic alcohol-fed male rats. Hepatology. 31:1135-1140.

29. Sudha, S., Balasubramanian, K., Arunakaran, J., and Govindarajulu, P. 1995. Preliminary study of androgen, thyroid and adrenal status in alcoholic men during deaddiction. Indian J. Med. Res. 101:268-272.

30. Jilka, R.L., et al. 1992. Increased osteoclast development after estrogen loss: mediation by interleukin-6. Science. 257:88-91.

31. O'Brien, C.A., Gubrij, I., Lin, S.C., Saylors, R.L., and Manolagas, S.C. 1999. STAT3 activation in stromal/osteoblastic cells is required for induction of the receptor activator of NF-kappaB ligand and stimulation of osteoclastogenesis by gp130-utilizing cytokines or interleukin1 but not 1,25-dihydroxyvitamin D3 or parathyroid hormone. J. Biol. Chem. 274:19301-19308.

32. Menaa, C., et al. 2000. Enhanced RANK ligand expression and responsivity of bone marrow cells in Paget's disease of bone. J. Clin. Invest 105:1833-1838

33. Yasuda, H., et al. 1998. Osteoclast differentiation factor is a ligand for osteoprotegerin/osteoclastogenesis-inhibitory factor and is identical to TRANCE/RANKL. Proc. Natl. Acad. Sci. USA. 95:3597-3602.

34. Kong, Y.Y., et al. 1999. OPGL is a key regulator of osteoclastogenesis, lymphocyte development and lymph-node organogenesis. Nature. 397:315-323.

35. Bellido, T., et al. 1995. Regulation of interleukin-6, osteoclastogenesis, and bone mass by androgens. The role of the androgen receptor. J. Clin. Invest. 95:2886-2895.

36. Pepersak, T., et al. 1992. Longitudinal study of bone metabolism after ethanol withdrawal in alcoholic patients. J. Bone Miner. Res. 4:383-387.

37. Diez, A., et al. 1994. Alcohol-induced bone disease in the absence of severe chronic liver damage. J. Bone Miner. Res. 9:825-831.

38. Johnell, O., Nilsson, B.E., and Wiklund, P.E. 1982. Bone morphometry in alcoholics. Clin. Orthop. 165:253-258.

39. Crilly, R.G., Anderson, C., Hogan, D., and Delaquerriere-Richardson, L. 1988. Bone histomorphometry, bone mass, and related parameters in alcoholic males. Calcif. Tissue Int. 43:269-276.

40. Laitinen, K., Lamberg-Allardt, C., Tunninen, R., Harkonen, M., and Valimaki, M. 1992. Bone mineral density and abstention-induced changes in bone and mineral metabolism in noncirrhotic male alcoholics. Am. J. Med. 93:642-650.

41. Kurihara, N., Bertolini, D., Suda, T., Akiyama, Y., and Roodman, G.D. 1990. IL-6 stimulates osteoclast-like multinucleated cell formation in long term human marrow cultures by inducing IL-1 release. J. Immunol. 144:4226-4230.

42. Tamura, T., et al. 1993. Soluble interleukin-6 receptor triggers osteoclast formation by interleukin-6. Proc. Natl. Acad. Sci. USA. 90:11924-11928

43. Girasole, G., et al. 1992. 17 $\beta$-estradiol inhibits interleukin- 6 production by bone marrow-derived stromal cells and osteoblasts in vitro: a potential mechanism for the antiosteoporotic effect of estrogens. J. Clin. Invest. 89:883-891.

44. Poli, V., et al. 1994. Interleukin-6 deficient mice are protected from bone loss caused by estrogen depletion. EMBO J. 13:1189-1196.

45. Roodman, G. 1992. Interleukin-6: an osteotropic factor? J. Bone Miner. Res. 7:475-478.

46. Gardner, J.D., Liechty, K.W., and Christensen, R.D. 1990. Effects of interleukin-6 on fetal hematopoietic progenitors. Blood. 75:2150-2155.

47. de la Mata, J., et al. 1995. Interleukin-6 enhances hypercalcemia and bone resorption mediated by parathyroid hormone-related protein in vivo. J. Clin. Invest. 95:2846-2852.

48. Rico, H., Cabranes, J.A., Cabello, J., Gomez-Castresana, F., and Hernandez, E.R. 1987. Low serum osteocalcin in acute alcohol intoxication: a direct toxic effect of alcohol on osteoblasts. Bone Miner. 2:221-225.

49. Laitinen, K., and Valimaki, M. 1991. Alcohol and bone. Calcif. Tissue Int. 49(Suppl.):S70-S73.

50. Klein, R.F., and Carlos, A.S. 1995. Inhibition of osteoblastic cell proliferation and ornithine decarboxylase activity by ethanol. Endocrinology. 136:3406-3411.

51. Klein, R.F., Fausti, K.A., and Carlos, A.S. 1996. Ethanol inhibits human osteoblastic cell proliferation. Alcohol Clin. Exp. Res. 20:572-578.

52. Giuliani, N., Girasole, G., Vescovi, P.P., Passeri, G., and Pedrazzoni, M. 1999. Ethanol and acetaldehyde inhibit the formation of early osteoblast progenitors in murine and human bone marrow cultures. Alcohol Clin. Exp. Res. 23:381-385.

53. Hurley, M.M., Martin, D.L., Kream, B.E., and Raisz, L.G. 1990. Effects of ethanol and acetaldehyde on collagen synthesis, prostaglandin release and resorption of fetal rat bone in organ culture. Bone. 11:47-51.

54. Chavassieux, P., Serre, C.M., Vergnaud, P., Delmas, P.D., and Meunier, P.J. 1993. In vitro evaluation of dose-effects of ethanol on human osteoblastic cells. Bone Miner. 22:95-103. 